\title{
Calidad Educativa en Educación Superior
}

\author{
Educational Quality in Higher Education \\ Qualidade Educacional no Ensino Superior
}

\section{ARTÍCULO GENERAL}

\author{
Dionisio J. Lunarejo López \\ dionisiolunarejo09@gmail.com \\ https://orcid.org/0000-0001-7801-8508 \\ Universidad César Vallejo, Lima - Perú
}

Recibido 07 de Abril 2021 | Arbitrado y aceptado 19 de Junio 2021 | Publicado en 03 Setiembre 2021

\section{RESUMEN}

Es importante conocer el estado en que se encuentra la calidad educativa en educación superior. Como Fundamento teórico, para el concepto de calidad educativa, encontramos que para Vásquez la noción de competencia tiene sus orígenes en el mundo empresarial, en el que se entiende como aquellas habilidades que tiene el trabajador para desarrollar su labor en una situación particular de manera rápida, adecuada $\mathrm{y}$ eficiente. $\mathrm{Al}$ respecto y de acuerdo con Aguerrondo es posible afirmar que la calidad es un concepto relativo que puede ser definido desde una perspectiva multidimensional. La calidad de una institución o de un programa se refiere a las características universales correspondientes a la educación superior en general, En las cuales va explicita la evaluación y el país tiene la obligación de garantizar que esa educación se entregue con calidad.

\section{ABSTRACT}

It is important to know the state of educational quality in higher education. As a theoretical foundation, for the concept of educational quality, we find that for Vásquez the notion of competition has its origins in the business world, in which it is understood as those skills that the worker has to develop their work in a particular situation quickly, adequate and efficient. In this regard, and according to Aguerrondo, it is possible to affirm that quality is a relative concept that can be defined from a multidimensional perspective. The quality of an institution or a program refers to the universal characteristics corresponding to higher education in general, in which the evaluation is made explicit and the country has the obligation to guarantee that this education is delivered with quality.

\section{RESUMO}

É importante conhecer o estado da qualidade educacional no ensino superior. Como alicerce teórico, para o conceito de qualidade educacional, constatamos que para Vásquez a noção de concorrência tem origem no mundo empresarial, no qual é entendida como aquelas competências que o trabalhador possui para desenvolver rapidamente o seu trabalho numa determinada situação., adequado e eficiente. A este respeito, e segundo Aguerrondo, é possível afirmar que qualidade é um conceito relativo que pode ser definido numa perspetiva multidimensional. A qualidade de uma instituição ou de um programa referese às características universais correspondentes ao ensino superior em geral, em que a avaliação é explicitada e o país tem a obrigação de garantir que esse ensino seja ministrado com qualidade. 


\section{Introducción}

A finales de la década de 1980 y en el transcurso de la siguiente década cuando los gobiernos de los países en América Latina prestan interés sobre la temática de la calidad educativa en el nivel superior y, al mismo tiempo, buscan rendir cuentas sobre el ejercicio del gasto público asignado en relación con el desempeño académico de las instituciones de educación superior (IES) (Claverie, González y Pérez, 2008; citado por Martinez, Tobon y Romero, 2017). Es importante conocer el estado en que se encuentra la calidad educativa en educación superior.

Como Fundamento teórico, para el concepto de calidad educativa, encontramos que para Vásquez (2015, citado por Martin, 2018) la noción de competencia tiene sus orígenes en el mundo empresarial, en el que se entiende como aquellas habilidades que tiene el trabajador para desarrollar su labor en una situación particular de manera rápida, adecuada y eficiente. Formulación que elimina de las prácticas educativas la formación crítica y creativa, porque reduce las competencias a procedimientos automatizados. Superando lo anterior Aguerrondo (1993, citado por Martin, 2018) plantea una serie de características y utilidad de dicho concepto, entre estas se encuentran: lo complejo y totalizante, donde se hace referencia al concepto de calidad como multidimensional, un segundo elemento es el social e históricamente determinado, el cual plantea la importancia de tener en cuenta las realidades específicas de un contexto determinado, un tercer elemento hace referencia a la transformación educativa, donde se hace necesario la redefinición del modelo en la toma de decisiones, un último elemento es el control de la eficiencia en el servicio, en esta parte se plantea la idea de la calidad educativa en el ajuste de decisiones con el fin de mejorar procesos. Al respecto y de acuerdo con Aguerrondo (1993) es posible afirmar que la calidad es un concepto relativo que puede ser definido desde una perspectiva multidimensional. La calidad de una institución o de un programa se refiere a las características universales correspondientes a la educación superior en general, En las cuales va explicita la evaluación y el país tiene la obligación de garantizar que esa educación se entregue con calidad.

El objetivo de la presente investigación es dar a conocer a los interesados, en qué estado se encuentra la calidad educativa. 
En relación a la inclusión educativa a nivel superior en Ecuador, Bell (2020), indica que:

El acceso formal a las instituciones ya no constituye un mecanismo satisfactorio de democratización ni inclusión y es necesario generar condiciones para la permanencia y egreso de los nuevos sectores sociales que acceden al nivel superior sin sacrificar la calidad y excelencia que - se supone - el mismo implica.

Luego describe las distintas fases que conforman el ciclo para la inclusión educativa en la educación superior como premisa para la promoción de la reflexión y de las transformaciones que respondan a las exigencias para su establecimiento y adecuado funcionamiento.

Otro factor importante de la calidad educativa en Educación Superior es la acreditación, sobre dicho aspecto, Martínez, Tobón y Romero (2017), afirmaron que: La acreditación es un proceso voluntario, en el cual la institución educativa se somete a la opinión de un organismo externo, con la finalidad de obtener un reconocimiento público de la calidad de su quehacer educativo (Universidad Nacional Mayor de San Marcos, 2009), siendo aplicable a las instituciones, como a los programas educativos. El proceso inicia con la autoevaluación institucional, seguido por la evaluación y opinión de pares académicos externos, y se finaliza con el informe de resultados sobre la acreditación de la calidad educativa (Contreras, 2012).

Actualmente, dichos procesos se dan mediante organismos públicos, privados o de naturaleza mixta, tanto de orden nacional como internacional

Al respecto, autores como Medina (2011) y Mondragón (2006), señalan que las políticas educativas en materia de educación superior siguen las recomendaciones emitidas por organismos internacionales, tales como: la Organización de las Naciones Unidas para la Educación, la Ciencia y la Cultura (UNESCO), la Organización para la Cooperación y el Desarrollo Económico (OCDE), el Banco Mundial (BM) y el Banco Interamericano de Desarrollo (BID), entre otros organismos que buscan que la educación se adapte a las exigencias planteadas por la sociedad.

Las IES están recurriendo a la acreditación de la calidad ante organismos externos, quienes tienden a abordar desde lo administrativo (Buendía, 2011b; Rangel, 2010), sin tocar la transformación de los procesos curriculares, las prácticas de 
aprendizaje, la evaluación, ni el emprendimiento de proyectos sociales. Además, se presentan problemas tales como: la simulación en los procesos de acreditación; la poca participación de los miembros de la comunidad educativa en los procesos de acreditación; la falta de aplicación del currículo por competencias. Es preciso estudiar estas problemáticas con el fin de implementar las mejoras continuas en los procesos académicos que lleven a formar profesionales que estén en condiciones de afrontar los retos actuales y futuros de la sociedad, algo que casi no está ocurriendo en las universidades acreditadas.

Por otro lado, la investigadora Brito (2018), nos presenta otro factor que se relaciona con la calidad educativa en educación superior, refiriéndose al síndrome de burnout en los profesores; indicando que:

La Organización Mundial de la Salud (2018) define la salud ocupacional como el conjunto de actividades asociado a disciplinas multidisciplinarias, cuyo objetivo es la promoción y mantenimiento del más alto grado posible de bienestar físico, mental y social de los trabajadores de todas las profesiones, promoviendo la adaptación del trabajo al hombre y del hombre a su trabajo. De acuerdo con diversos estudios realizados, los profesionales más propensos a presentar estrés en sus trabajos son los técnicos de la salud y los profesores (Ferrel, 2013)

Cárdenas, Méndez y González (2014) conciben a los docentes de nivel superior como parte fundamental para lograr la calidad educativa en las Instituciones de Educación Superior (IES).

El síndrome de burnout también se conoce como el síndrome de desgaste profesional. Es un estado de cansancio físico y emocional que surge como consecuencia de la sobrecarga laboral (Ortega y López, 2004).

En este sentido, se considera relevante realizar una investigación que mida tanto la satisfacción de los profesores hacia su trabajo, como el agotamiento, desinterés, desánimo y posible depresión en los profesores de una institución de educación superior.

El objetivo general es identificar la propensión a padecer síndrome de burnout en los docentes de una IES. Los objetivos específicos son los siguientes: 1. Evaluar el nivel de agotamiento por sobrecarga y exceso de trabajo. 2. Evaluar el nivel de cinismo al 
hacer solo lo mínimo en el trabajo. 3. Evaluar el nivel de eficacia profesional con base en la carencia de logros y productividad en el trabajo.

El propósito de la investigación es realizar un caso de estudio sobre la propensión de padecer el síndrome de burnout, ya que diversos estudios del tema aplicados en el sector educativo han demostrado que el profesorado parece ser especialmente vulnerable a este problema, debido a la presión que soportan los profesores al intentar responder a la diversidad de demandas.

\section{Método}

En la investigación: Comprensión del ciclo para la inclusión educativa en la educación superior ecuatoriana; El enfoque es predominantemente cualitativo, desarrollada entre el mes de marzo de 2016 y agosto de 2017, que se ajusta a los criterios generales de la investigación acción participativa que comprende la realidad social y educativa como una configuración total y compleja, supone reflexión y acción y está orientada a una mejor comprensión de la práctica educativa para su transformación (Gómez y Roquet, 2012). Desde esa perspectiva, en la investigación se cumplieron las etapas señaladas por Martí (2000), es decir: pre-investigación, diagnóstico, programación y conclusiones y propuestas. Mientras que en la investigación:

Problemáticas relacionadas con la acreditación de la calidad de la educación superior en América Latina; el tipo de estudio: Se efectuó una investigación cualitativa sobre las problemáticas relacionadas con la acreditación de la educación superior en América Latina. Por medio de una investigación documental a partir de la revisión bibliográfica sobre lo que se ha investigado, escrito y publicado en relación con el tema (Del Cid, Mendez y Sandoval, 2011). El análisis de la información se hizo mediante la técnica del fichaje.

Categorías de análisis: Según los documentos revisados, se detectaron las principales problemáticas que enfrentan las IES en los procesos de acreditación de la calidad; cada problemática se consideró una categoría de análisis: a) Problemas respecto a la metodología y modelo general de la acreditación; b) Problemas de la acreditación por la simulación en los procesos; c) Problemas de la acreditación por el incremento del aparato burocrático en las IES; d) Problemas de la acreditación por el grado de participación de los miembros de la comunidad educativa; e) Problemas de la 
acreditación en torno a la credibilidad en los organismos externos; f) Problemas de la acreditación en torno a la aplicación del currículo por competencias en las IES; g) Problemas de la acreditación en torno a la finalidad de las IES.

\section{Desarrollo y discusión}

En relación al artículo "Problemáticas relacionadas con la acreditación de la calidad de la educación superior en América Latina" de los autores: Martínez, J., Tobón, S., y Romero, A. (2017). Cada problemática encontrada se consideró una categoría de análisis:

\section{Problemas respecto a la metodología y modelo general de la acreditación}

La metodología y el modelo de acreditación de las IES tiende a enfocarse demasiado en los procesos administrativos (Buendía, 2011b; Rangel, 2010). Han descuidado la evaluación de desempeños en la formación de profesionales y el cambio social (Buendía, 2011b; Salas, 2013). Esto se aprecia en los indicadores e instrumentos de organismos acreditadores de América Latina. Quienes se enfocan más en lo administrativo que en evaluar el impacto en el entorno.

Si se quiere impulsar un sistema de mejora continua y la implementación de una verdadera cultura de calidad en las IES que implique la transformación de las condiciones sociales, es necesario, como señalan Martínez, Mateo y Reyes (2011), incorporar cambios en los sistemas de acreditación, buscando que se entren más en el desempeño de las universidades, las unidades académicas, los programas y los diferentes actores (estudiantes, docentes y directivos), y menos en tareas procedimentales, de tal manera que contribuyan a cambiar las prácticas educativas tradicionales centradas en contenidos y en la fragmentación de las asignaturas.

Ante esto, los indicadores que establecen los organismos acreditadores además de enfocarse en procesos y procedimientos, deben acompañarse de evidencias puntuales que describan resultados significativos de las IES (Herrera y Aguilar, 2009), como la disminución de la deserción, aumento de la eficiencia terminal, logro del perfil de egreso, grado de empleo de los egresados, proyectos de emprendimiento, proyectos de innovación, patentes, publicación de artículos en revistas indexadas, esto tendría impacto en las funciones sustantivas de las universidades. 


\section{Problemas de la acreditación por la simulación en los procesos de evaluación}

Para obtener recursos públicos, las IES deben de buscar la acreditación de su calidad, los actores involucrados recaen en la simulación de procesos con la intención de presentar indicadores que reflejen una buena calidad por parte de la institución educativa, acomodando los documentos con lo esperado, sin que sean reales (Rangel, 2010).

La simulación tiende a darse respecto a procesos académicos tales como: 1) productividad académica, colocando como publicaciones la presentación de conferencias, ponencias y charlas en diversos eventos; 2) recogiendo evidencias a último momento, como listas de asistencias, asistencia a eventos, organización de eventos, aplicación de encuestas a estudiantes, otorgamiento de becas; 3) contratación de investigadores de alto nivel solo para demostrar que se tiene y mejorar los índices de publicaciones y de talento humano especializado; 4) eliminación de datos negativos para no afectar los índices de deserción.

\section{Problemas de la acreditación por el incremento del aparato burocrático en las IES}

Con la finalidad de buscar acreditar la calidad educativa, se han creado áreas encargadas de llevar acabo los procesos de evaluación y seguimiento de la calidad. Buendía (2011b, 2013) señala que, con esta burocratización, ocurre una desarticulación entre los aspectos académicos y los administrativos, en donde los resultados obtenidos en procesos de evaluación no son comunicados a toda la comunidad educativa de las IES. Esto se debe, a la falta de tiempo de los responsables de la autoevaluación por la gran cantidad de formatos que deben completar para la acreditación.

\section{Problemas de la acreditación en torno al grado de participación de los miembros de la comunidad educativa de las IES}

Muchas veces los modelos de acreditación en Latinoamérica no establecen con indicadores puntuales la participación de todos los actores educativos en la cultura de la calidad, por lo cual este proceso termina siendo dirigido por una sola persona o equipo (Urbano, 2007). Aunado a esto, las IES carecen en muchos casos de políticas 
institucionales que fomenten la participación de los diferentes actores en el mejoramiento de la calidad educativa (Ovando, Elizondo y Grajales, 2015).

Debe de involucrarse a los docentes como protagonista de este proceso, ya que no puede haber calidad académica si las prácticas en el aula continúan centradas en formar para la sociedad feudal o industrial, y no para la sociedad del conocimiento (Gómez, Zamora y Torres, 2014).

\section{Problemas de la acreditación en torno a la credibilidad en los organismos externos}

En la práctica, los procesos que se llevan a cabo no necesariamente son sinónimos de calidad educativa, y menos cuando no se considera el impacto en las funciones sustantivas de las universidades (Gregorutti y Bon, 2013). Algunas de las causas por las cuales se cuestiona la credibilidad de los organismos internacionales son: 1) los procesos son extensos y descontextualizados (Uribe, 2013); 2) no se evalúan productos de desempeño; 3 ) falta ética y transparencia en los procesos de evaluación; 4) se quedan en completar muchos formatos; y 5) las evaluaciones son complicadas y caen demasiado en lo formal.

Los organismos acreditadores deberían tener un proceso de formación en lo que significa calidad educativa, para que no solo se dediquen a llevar una lista de cotejos de las actividades realizadas, sino que evalúen el impacto en la formación de los ciudadanos, la productividad en investigación, el desarrollo tecnológico y el emprendimiento para mejorar las condiciones de vida de la sociedad.

\section{Referencia}

Bell, R. (2020). Comprensión del ciclo para la inclusión educativa en la educación superior ecuatoriana. Revista electrónica Educare. EISSN: 1409-4258 Vol. 24(1) Enero-Abril, 2020: 1-21. Doi: http://doi.org/10.15359/ree.24-1.4. URL: http://www.una.ac.cr/educare

Martínez, J., Tobón, S., y Romero, A. (2017). Problemáticas relacionadas con la acreditación de la calidad de la educación superior en América Latina. Innovación educativa (México, DF), 17(73), 79-96. Recuperado en 01 de diciembre de 2020. http://www.scielo.org.mx/scielo.php?script=sci arttext\&pid=S1665-

\section{$\underline{26732017000100079 \& \operatorname{lng}=\mathrm{es} \& \operatorname{tlng}=\mathrm{es}}$}


Universidad Nacional Mayor de San Marcos. (2009). Calidad educativa y certificación profesional. Compendio: autoevaluación, evaluación externa, acreditación y certificación. Lima, Perú: Universidad Nacional Mayor de San Marcos/Oficina Central de Calidad Académica y Acreditación.

Contreras, F. (2012). Evaluación, acreditación de carreras y bibliotecas de educación superior. Lima, Perú: Colegio de Economistas del Callao.

Medina, S. R. (2011). Los organismos internacionales y la evaluación como política educativa en México: elementos para un balance. En S. R. Medina, Políticas y educación. La construcción de un destino (págs. 17-38). México, D. F.: Ediciones Díaz de Santos/UNAM.

Mondragón, A. R. (2006). La acreditación y certificación en las instituciones de educación superior. Hacia la conformación de circuitos académicos de calidad: ¿exclusión o integración? Revista del Centro de Investigación, 7(26), 51-61.

Buendía, A. (2011b). Evaluación y acreditación de programas en México. Más allá de los juegos discursivos. Diálogos sobre Educación, 2(3). Recuperado de http://www.revistadialogos.cucsh.udg.mx/sites/default/files/dse_a2_n3 juldic2011 buendia.pdf

Rangel, H. (2010). Hacia una evaluación generadora. Más allá de la evaluación tecnicoburocrática de las universidades en México. Revista Iberoamericana de Educación, 54(1). Recuperado de: http://rieoei.org/deloslectores/3794Rangel.pdf

Del Cid, A., Méndez, R., y Sandoval, F. (2011 ). Investigación. Fundamentos y metodología. México. 2da. edición: Pearson.

Brito J. (2018). Calidad educativa en las instituciones de educación superior: evaluación del síndrome de burnout en los profesores. Revista Iberoamericana para la Investigación y el Desarrollo Educativo ISSN 2007- 7467 Vol. 8, Num. 16 EneroJunio 2018 DOI: 10.23913/ride.v8i16.356

Ferrel, R. (2013). El síndrome de quemarse en el trabajo (burnout) en docentes universitarios. Duazary: Revista Internacional De Ciencias De La Salud, 7(1), 15 - 28. DOI:10.21676/2389783X.306. 
Cárdenas, M., Méndez, L. y González, M.T. (2014). Desempeño, estrés, Burnout y variables personales de los docentes universitarios. EDUCERE, 18(60), 289-302. DOI: https://doi.org/10.15517/aie.v14i1.13210.

Ortega, C. y López, F. (2004). El Burnout o síndrome de estar quemado en profesionales sanitarios: Revisión y perspectivas. International Journal of Clinical and Health Psychology, 137-160.

Claverie, J., González, G., y Pérez, L. (2008). El sistema de evaluación de la calidad de la educación superior en la Argentina: el modelo de la CONEAU. Alcances y limitaciones para pensar la mejora. Revista Iberoamericana de Evaluación Educativa, 1(2), 148-164.

Martin, J. (2018) Calidad educativa en la educación superior colombiana: una aproximación teórica. Sophia, 14(2), 4-14. Universidad la gran Colombia DOI: http://dx.doi.org/10.18634/sophiaj.14v.2i799

Rangel, H. (2010). Hacia una evaluación generadora. Más allá de la evaluación tecnicoburocrática de las universidades en México. Revista Iberoamericana de Educación, 54(1). Recuperado de: http://rieoei.org/deloslectores/3794Rangel.pdf

Buendía, A. (2011b). Evaluación y acreditación de programas en México. Más allá de los juegos discursivos. Diálogos sobre Educación, 2(3). Recuperado de http://www.revistadialogos.cucsh.udg.mx/sites/default/files/dse_a2_n3 juldic2011 buendia.pdf

Buendía, A. (2013). Genealogía de la evaluación y acreditación de instituciones en México. Perfiles Educativos, 35(número especial), 17-32.

Urbano, N. (2007). Efectos de la implementación del modelo colombiano de acreditación de programas académicos. Un análisis a partir del caso de los programas tecnológicos. Universitas Humanística, 64, 139-161.

Ovando, M., Elizondo, M., y Grajales, O. (2015). La evaluación y la acreditación desde la perspectiva de los universitarios: una experiencia educativa en la Universidad Autónoma de Chiapas. Revista de Sistemas y Gestión Educativa, 2(4), 936-944. 
Gómez, E. A., Zamora, R., y Torres, J. (2014). Los proceso de acreditación en la

Universidad Autónoma de Chapingo: situación actual. Ponencia presentada en el Congreso Iberoamericano de Ciencia, Tecnología, Innovación y Educación, Buenos Aires, Argentina. Recuperado de:

http://www.oei.es/congreso2014/memoriactei/98.pdf

Gregorutti, G. J., y Bon, M. V. (2013). Acreditación de la universidad privada ¿Es un sinónimo de calidad?. Revista Iberoamericana sobre Calidad, Eficacia y Cambio en Educación, 11(1), 122-139.

Uribe, A. P. (2013). Los factores que conforman el modelo de acreditación de alta calidad de programas académicos en Colombia, revisión desde el enfoque de la percepción. Revista de la Facultad de Ciencias Económicas, Administrativas y Contables, 5(2). Recuperado de: http://publicaciones.unisimonbolivar.edu.co:82/rdigital/ojs/ index.php/desarrollogerencial/article/view/202/198 